\title{
Reflecting the evidence
}

\author{
Derek Richards \\ Editor, Evidence-based Dentistry
}

Evidence-Based Dentistry (2008) 9, 98. doi: 10.1038/sj.ebd.6400607

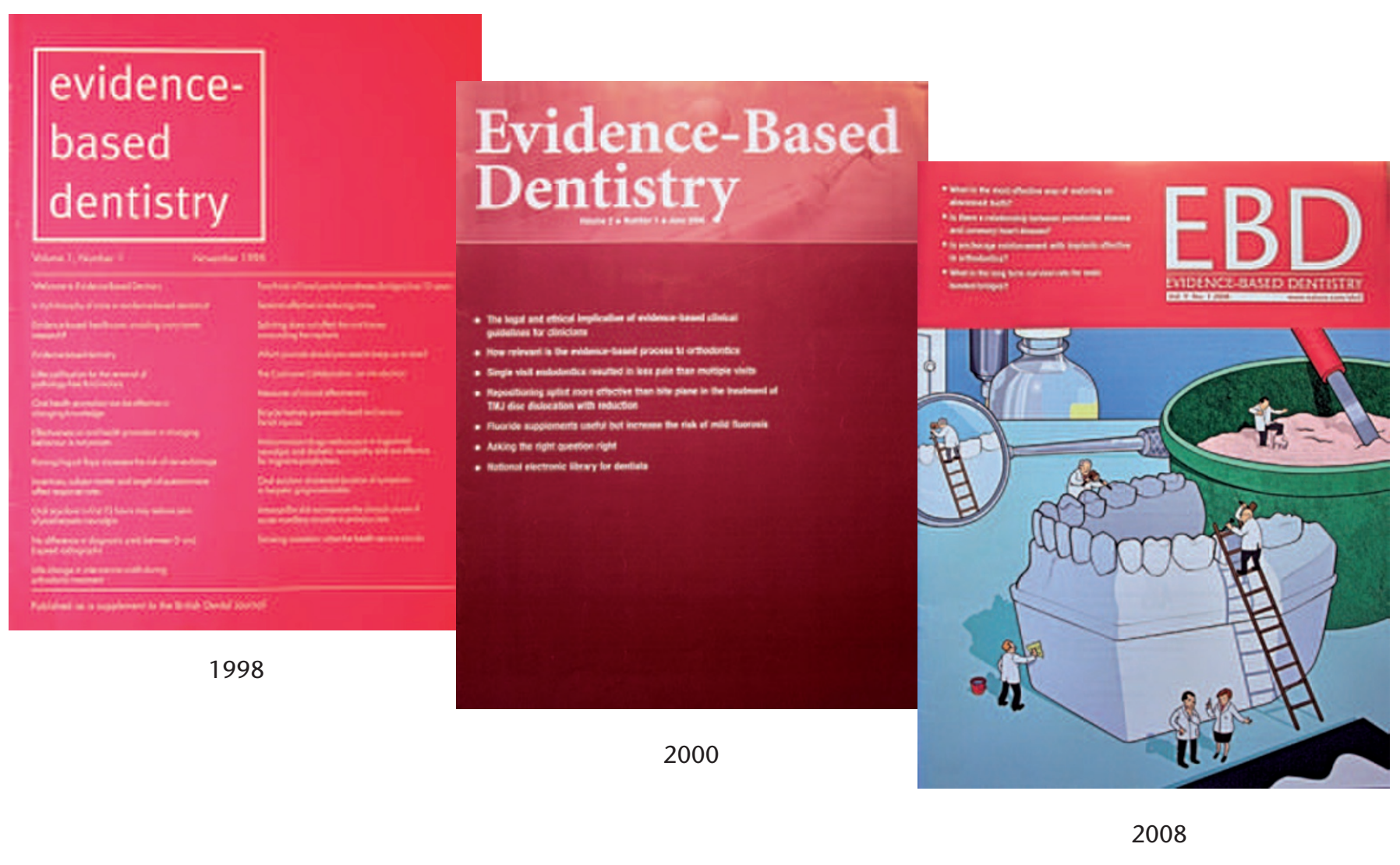

Writing at the end of November 2008 it is interesting to reflect that it is now ten years since the first issue of Evidence-based Dentistry was published as a supplement of the BDJ. Since then the journal has grown, moving from a biannual supplement to a quarterly stand-alone journal in 2000. Development of the journal led to it achieving a Medline listing in 2004. As with all publications the journal has, I believe, improved over the years, with perhaps some of the more noticeable changes being the changing cover style (above).

The main aim of the journal has always been to provide a dissemination route for high quality research relevant to dental practitioners. This we endeavour to do through our summaries of research and evidence-based guidelines which remain the core of the journal. Recently we have introduced the Dental Evidence-based Topic or DEBT: these are short critical summaries of particular clinical topics or questions which aim to provide a more detailed analysis of an area than our standard summaries of single studies or reviews.

The journal's other aim has been to provide tools and information to enable practitioners to practise evidence-based dentistry. These are found in the Toolbox section of the journal and have covered a range of articles on statistics, web and papers based resources. We have also included pri- 
mary research related to evidence-based methodologies in dentistry.

None of this would be possible without the small core editorial team who work hard to keep the publication on track, and the support of our commentators. The support of the commentators is also vital to EBD and in these increasingly busy times it is rewarding that they are willing to undertake this work, with the publication as their only reward. For their support I am very grateful and I am hopeful that with this continued support we will celebrate our $20^{\text {th }}$ year in 2018 .

Continuing the reflective mood, this journal has long used a hierarchy of evidence to rate our commentaries (see page 108). As regular readers will be aware this is derived from the Centre for Evidencebased Medicine levels of evidence document (www.cebm.net/index.aspx?o=1025). In the recent Harveian Oration at the Royal College of Physicians (www.rcplondon. ac.uk/news/news.asp?PR_id=422 ) Professor Sir Michael Rawlins, Chair of the National Institute for Health and Clinical Excellence (NICE) argued for a new approach to analyzing clinical evidence. He claims that randomised controlled trials (RCT) have been put on an underserved pedestal and that their position at the top of hierarchies is inappropriate and that hierarchies are illusory tools for assessing evidence and should be replaced by a diversity of approaches that involve the totality of the evidence-base.

RCTs have a number of well known limitations; they are impossible for very rare diseases, prophylactic antibiotics for infective endocarditis being a good example. There are unnecessary when treatments produce dramatic benefit. RCTs are expensive and there are also issues about their generalisability. Young and Godlee ${ }^{1}$ in their editorial were of the opinion that Michael Rawlins' central question was not what we should do instead of randomised controlled trials, for RCTs continue to produce valuable information that can improve health, but how policy makers, resource controllers and health care workers can use the full spectrum of research to improve health, that is important. I agree with this view, and the recent Canadian guideline on bisphosphonate associated osteonecrosis of the jaw which we summarise on page 101 is a good example of where a multidisciplinary group have used all the available evidence in order to provide guidance on how we should manage an emerging problem. A rare problem, it is unlikely that an RCT will take place in the near future so we need to summarise all the available evidence in a robust manner in order to help patients and clinicians make informed decisions about their care. To me this is what evidence-based practice has always been about as originally defined by Sackett et al, 'the conscientious, explicit and judicious use of best evidence in making decisions about care of individual patients'. ${ }^{2}$ In the early days of the evidence-based health care movement there was undoubtedly a great deal of focus on the value of the RCT, but as Sackett and Wennberg ${ }^{3}$ pointed out early on, the type of research we need to do depends on the question we are trying to address and we should use the best research design for each questions.

In reality many health problems are complex and cannot be resolved by simple interventions and require complex interventions. In 2000 the Medical Research Council proposed an evaluation framework for complex interventions ${ }^{4}$. This has now been completely revised and updated and can be downloaded from the MRC website (www. mrc.ac.uk/Utilities/Documentrecord/index. htm?d=MRC004871), while Craig et al ${ }^{5}$ have summarized the issues and key messages in the new guidance in an article in the British Medical Journal.

Evidence-based practice has certainly developed in the ten years since the launch of the journal and the phrase evidencebased is now liberally spread through journals, presentations and policy documents. However, I feel that it is often over used and misused and this provides its detractors, for there are still many, with ammunition to rubbish the original concepts and ideas. There is clearly a long way to go to both improve our understanding of the available evidence to support health care interventions and to improve our understanding of the best methods to ensure their uptake and implementation in order that we can best serve our patients. Until we have mastered these challenges there is still a clear role for this journal in disseminating the best available evidence to the profession.

1. Young C, Godlee F. The BMJ Evidence Centre. BMJ. 2008 Nov 7;337:1126.

2. Sackett DL, Rosenberg WM, Gray JA, Haynes RB, Richardson WS. Evidence based medicine: what it is and what it isn't. BMJ. 1996 Jan 13;312:71-72.

3. Sackett DL, Wennberg JE. Choosing the best research design for each question. BM]. $1997 \mathrm{Dec}$ 20-27;315(7123):1636.

4. Medical Research Council. A framework for the development and evaluation of RCTs for complex interventions to improve health. London: MRC, 2000.

5. Craig P, Dieppe P, Macintyre S, Michie S, Nazareth I, Petticrew M; Medical Research Council Guidance. Developing and evaluating complex interventions: the new Medical Research Council guidance. BMJ. 2008 ;337: 979- 983 